\title{
Emigración potosina en el siglo XVIII: el linaje Díaz en Calbuco
}

Pablo A. Pérez

Investigador independiente

\begin{abstract}
The study documents the presence of an emigrant from the city of Potosí, who was established in the southern Spanish colonial possession of Calbuco (belonging to the orbit of Chiloé), in the first half of the eighteenth century. The lineage originated by him appears in two different branches, providing genealogical and historical materials on both locations. Within a period of almost two centuries, there are approximately thirty people documented that are linked to the male lineage.
\end{abstract}

Keywords

Calbuco, Chiloé, Díaz, genealogy, migration, Potosí

\section{Resumen}

El trabajo documenta la presencia de un emigrante natural de la ciudad de Potosí, establecido en la austral posesión colonial española de Calbuco (perteneciente a la órbita de Chiloé), en la primera mitad del siglo XVIII. EI linaje por él formado se presenta en dos distintas ramas, aportando material genealógico e histórico sobre ambas localidades. En un período de casi dos siglos se documentan alrededor de una treintena de personas vinculadas a la estirpe por vía de varón. 
Palabras claves

Calbuco, Chiloé, Díaz, emigración, genealogía, Potosí

\section{Introducción}

No existen referencias acerca de emigrantes potosinos establecidos en las zonas de influencia de Chiloé durante el período colonial. Este trabajo constituye una corta noticia acerca de un personaje de este origen presente en la primera mitad del siglo XVIII en Calbuco, lugar este último perteneciente a la órbita cultural, histórica y política de Chiloé.

Luego de algunas breves noticias sobre la situación del Potosí y de Calbuco de antaño, se presenta la tabla de genealogías confeccionada a partir de varios libros parroquiales.

\section{El entorno potosino}

La antigua Villa Imperial de Potosí se encuentra a más de 4.000 metros sobre el nivel del mar, a la falda del legendario Cerro Rico, que alberga la mina de plata más grande y famosa del mundo (Taullard 25). Potosí dispone de toda una fraseología elogiosa, que la condecora como "Ciudad Única" o "La Campana de Plata", y también se la exorna con otros títulos con cuyo brillo se ha querido realzar la belleza espiritualizada de la ciudad de excelsitud histórica (Otero 469).

El origen de la villa y su desarrollo tienen estrechísima ligazón con la minería. De hecho, el régimen de mita fue utilizado para esta explotación desde 1573 hasta incluso 1825 (Cole 1). La mita fue el detonador de un proceso económico y social de bastante importancia (Salazar-Soler 147). La población de la localidad se componía de abundante población indígena y los mitayos destinados al trabajo en las minas vivían en barrios específicos (cada uno de ellos, con parroquia, alcalde y un cacique), divididos de acuerdo a su procedencia (Gisbert 251). Dice un historiador de fines del XVIII a propósito de Potosí:

Provincia y Gobierno del Reyno del Perú en el Arzobispado de Charcas, fué antes Corregimiento, confina su jurisdiccion por el Curato de Salinas con la Provincia de Paria que está al N E, y todo lo demas de su distrito la rodea la de Porco; es país montuoso, lleno de cerros, riscos y quebradas, de temperamento muy frio, y por eso estéril de frutos, tiene 10 leguas de 
largo E O y 7 de ancho; en su territorio se cria mucho ganado ovejuno, carneros de la tierra y algunas vicuñas, tiene muchas minas de sal cristalina; baña esta Provincia el rio Pilcomayo á donde van á parar muchos arroyos, sus habitantes llegan á 25ə. La Capital tiene el mismo nombre.

Villa fundada el año de 1545 á la falda del monte que tambien se llama así, y de una quebrada por donde pasa un riachuelo que la riega, se formó del curso de gente que se juntaba al trabajo de la mina (Alcedo IV, 289).

Y prosigue más adelante de esta manera, refiriéndose a la composición de sus poblaciones:

[...] tenia 19 Curatos en la Villa y su distrito, de los quales 14 se reduxeron el año de 1759 á siete con los nombres de la Matriz que es de Españoles, y para Indios San Pablo y San Sebastian, San Juan y San Martin, la Concepcion y San Christóval, San Pedro y San Francisco, Santiago y Copacavana, San Benito y Santa Bárbara, San Bernardo y San Lorenzo, y San Roque, en su inmediacion hay unos baños calientes medicinales muy acreditados que llaman de Don Diego (Alcedo 289-290).

El cerro de Potosí, con una enorme demanda de productos alimenticios en razón de la concentración poblacional, unido a la especialización en las labores mineras y a estar ubicado en los desolados páramos altoandinos, reclamaba un volumen de granos muy importante (en 1603, por ejemplo, la Villa Imperial de Potosí demandaba 50.000 fanegas de trigo anuales y cerca de 100.000 de trigo) (Pérez Herrero 129).

Ya en el siglo XVII consta que "Es el Potosí el lugar de más gente del reino, así de españoles como naturales" (Ordóñez de Ceballos 453). Sobre el sitio también se escribe lo siguiente:

Su situación es incómoda, por ser algo pendiente y de irregular formación; su temperamento sano, aunque muy frío en todo tiempo, y faltando en él oportuno el calor, principio de la vegetación, nada produce su suelo en muchas leguas de distancia. Es paso y garganta precisa para todo el Perú, debiéndole considerar como el Cádiz de este reino, según la abundancia de gente que viene a sus negocios por causa de la mita, y demás trabajos e incidencias del mineral (Pino Manrique 5). ${ }^{1}$

A inicios del 1700 los españoles no llegaban a 3.000 vecinos, aunque el número de moradores cambia, ya que entran unos y salen otros; y el número total entre indios y españoles rondaba los 70.000, habitando en unas 16.000 casas (Arzáns de Orsúa y Vela 26-27).

\footnotetext{
1 Juan del Pino Manrique fue el primer gobernador intendente de la provincia de Potosí.
} 
Esta cifra parece muy acertada, ya que en 1719 Potosí tenía 60.000 moradores, de los cuales 35.000 eran indios (Navarro García 654); luego, en los años 1719-1720, la ciudad fue asolada por una peste que se llevó "infinitos Indios Mitayos" (Alcedo 290), en número de 22.000 (Navarro García 654), y otros tantos abandonaron la ciudad. La primera mitad del siglo XVIII fue para Potosí -y para toda la minería peruana- una época de decadencia que alcanzaba allí un punto culminante, reflejado en la producción argentífera (Navarro García 664-65; Mira Delli-Zotti 286); y desde mediados de la década de 1730 hasta la década de 1790, se define una tendencia de alza modesta (Tandeter 13).

En cuanto al entorno geográfico de la Villa, dice un cronista: "Lugares desolados sin orden ni formación; casas pajizas derramadas por los campos; total abandono de estos; ríos sin puentes; caminos casi intransitables; ninguna comodidad para los viajes; distancias muy largas sin albergue, y gentes sin cultura, es la fea imagen que presenta cada uno de estos países más o menos cercano o distante a los caminos reales o pueblos numerosos" (Pino Manrique 8).

El territorio sufrió a comienzos del siglo XIX una aguda crisis minera, a la que la azoguería potosina colaboró con la fragmentación política de los territorios españoles americanos. Hay registro de emigrantes bolivianos asentados en territorio de Chile, en número de 133 para 1854, 192 para 1865, 278 para 1875, 13.146 para 1885, 8.669 para 1895, 21.968 para 1907, etc. (Naciones Unidas, Cuadro 62.C. "Extranjeros residentes"). En tiempo contemporáneo, se señala un aspecto negativo para la estabilidad demográfica potosina, que es la permanente migración de sus habitantes al interior y exterior del país, siendo la principal causa la falta de fuentes de trabajo (Tapia Montecinos et al. 31). Esta dinámica migratoria sigue en la actualidad en vigencia (PNUD 68).

\section{Calbuco}

Chile mantuvo relaciones comerciales con Potosí en tiempo colonial. Un cronista de aquel entonces es específico cuando escribe:

Del reino de Chile le traen hermosos caballos, siendo éstos de tan aventajado brío y gallardía que en muchos años de los pasados se compraron por 2,000 pesos, y hoy se compran muy baratos. Son en bondad tan excelentes que quien tiene experiencia de unos y otros aseguran no excederles los de Andalucía. También de la villa de Cochabamba y sus amenísimos contornos le traen otros galanos y briosos 
caballos, moderados en el precio. Aventájase a más el reino de Chile, pues además de darle tan veloces y bizarros caballos, también le da ricos, curiosos y apreciados barros, cordobanes, almendras, arroz, anís, orégano, mostaza, nueces y otras cosas necesarias para la vida humana (Arzáns de Orsúa 22).

De la zona de Chiloé no hay noticia concreta de relaciones comerciales con Potosí. Por ejemplo, se da cuenta de los excesos del presidente D. ${ }^{n}$ Juan Andrés de Ustáriz, que envió a la provincia de Chiloé a D. ${ }^{n}$ Alejandro Garzón de Garaicoechea, criado suyo, como capitán de Calbuco el año de 1710, en ausencia y por enfermedad del general nombrado por Su Majestad (Marín de Velasco) "solo para tenerlo de cajero y comerciante de los géneros y ropas de dicha provincia la que se le enviaban crecidas porciones de ponchos, sobrecamas y otros tejidos para remitirlos á Potosí, donde se vendían al crecido precio que allí alcanzaban, encargándose de llevar estas especies don Juan Felipe Valladolid, otro criado de Ustáriz" (Silva y Molina Libro IV, 22).

San Miguel de Calbuco, bañado en las azules aguas del Pacífico y perteneciente a la historia y cultura de Chiloé, era en los albores del 1700 un fuerte instalado en la isla de Caicaén y poseía un puerto donde podían atracar bastantes embarcaciones. El partido de San Miguel de Calbuco estaba compuesto en el siglo XVIII por:

[...] las diez islas siguientes: Abtao (es la mas occidental), Quigua, Caicahen (en ella está el fuerte), Chaullin, Puluqui (es la mayor), Guar (es la mas oriental), Mailen (es la mas setentrional), Chidguapi, Quenu y Tabon, que es la mas meridional. Todas son apropósito para el cultivo i lo están bastante, con poblacion proporcionada al resto de la provincia, menos Mailen i Chaullin, que están desiertas. Tabon, Caicahen, Quenu i Chidguapi carecen de leña, pero son fértiles i sus playas abundan de toda suerte de marisco excelente, i en esto, con particularidad el bajo de Lameguapi o isla de los Lobos, que está al sur de Quenu i Caicahen, i se descubre mucho a bajamar, formando 4 o 5 isletas rasas, con algunas rocas (Moraleda i Montero 138-39).

$[\ldots]$

El terreno de las islas dichas es bastante apropósito para cultivo i efectivamente lo está al respecto del sistema general de la provincia, e igualmente en punto a poblacion; no así la tierra firme vecina, porque aunque su terreno es en muchas partes ventajoso para tal efecto, prefieren estas jentes la habitacion en islas, tanto por el grande ausilio del marisco que prestan sus playas (alimento de primera necesidad en toda la provincia), como por libertarse o defenderse mejor en ellas de las irrupciones que han solido hacer los indios juncos y llanistas de Osorno en este partido (Moraleda i Montero 192-93). 
La gran riqueza maderera llevaba a convertir este comercio en el principal ramo de exportación de la provincia. Sobre esta granjería se relata lo siguiente:

Los astilleros (así llaman a los sitios donde hacen los cortes) mas frecuentados i de donde sale el mayor número de tablas están en la jurisdiccion de Calbuco, I son Contao, el estero de Coitue, el rio de Coihuin, Cayenel i la manga distante de 8 a 9 leguas del fuerte de Calbuco i de 22 a 24 de este puerto de San Carlos, esto es por lo que hace a navegacion, a que se deben agregar 6 a 8 mas que hai desde la orilla del mar hasta el lugar donde hacen las tablas, que conducen al hombro por un terreno áspero, quebrado i con frecuentes precipicios, que solo les permiten cargas casi la mitad de lo que pudieran por un terreno i camino regular (Moraleda i Montero 216).

Además, Calbuco adquiría mucha importancia en todo lo que se relacionaba a los viajes que se realizaban a la vertiente oriental de los Andes: las expediciones esclavistas o malocas, las misiones evangelizadoras y las entradas exploradoras tenían por puerto de origen al estratégico puerto de Calbuco (Arias 8). En el abanico de emigrantes llegados a estas australes latitudes, se cuenta ahora con un personaje altoperuano, cuyas informaciones y descendencia son presentadas a continuación.

\section{Tabla de descendencia}

Isidro Díaz es el emigrante dieciochesco que, oriundo de Potosí, contrajo matrimonio en 1747 en la localidad de Calbuco. Por desgracia, no queda constancia documental de los padres de los novios, por lo que la filiación de Isidro Díaz presenta algunos problemas.

Su nacimiento se estima alrededor de 1720 (aunque pudiera ser bastante anterior a esta fecha) y no ha podido ser encontrada su fe de bautismo, a pesar de haberse consultado los registros bautismales de la parroquia de Santiago Apóstol de Potosí desde 1680 en adelante. Existe constancia de algunos bautismos de niños naturales que recibieron el nombre de Isidro, entre los cuales alguno podría ser la persona buscada, ${ }^{2}$

\footnotetext{
2 Las personas que más posibilidades tienen de ser el Isidro Díaz sobre quien se escribe en este trabajo son:

- Isidro. Hijo de Magdalena Carrizales, soltera. Bautizado de un año y nueve meses, el 14/2/1695 en Potosí, siendo sus padrinos Francisco de Otalora y Josefa Muñoz (LBPs9, foja 98).

- Isidro. Hijo natural de Catalina Figueroa y de padre no conocido. Bautizado de dos días de edad el 18/5/1718 en Potosí, siendo su padrino D. " Sebastián Domingo de Lusa y María Santos (LBPs3, foja 223v).
} 
aunque también cabe la posibilidad de que hubiera nacido en alguna villa de las cercanías de Potosí.

Isidro Díaz está registrado como zapatero (1767), unos años después de contraer matrimonio (1747) con una señorita del linaje Mejía establecido en Calbuco. La descendencia de los consortes (que consta a veces relacionada a la localidad costera de Caicaén, en el extremo sur de la antigua isla de Calbuco) se extiende hasta casi el año 1900, aunque es sumamente probable que la misma se prolongue por varonía hasta el día de hoy.

Los Díaz potosinos entroncaron con algunas familias coloniales típicas (como los Oyarzun, los Hernández, los Guerrero, los Silva o los Soto), aunque también hay uniones con indígenas (como los Maricán), algunas de ellas naturales.

\section{Tronco del linaje}

I. Isidro Díaz. Consta como natural de la ciudad de Potosí en ocasión de atestiguar en un casamiento (8/8/1757 en el fuerte de San Miguel de Calbuco ${ }^{3}$ ). Tal vez es el mismo Isidro que es consignado como zapatero (25/3/1767 en el fuerte de San Miguel de Calbuco). ${ }^{4}$ Sin duda debe ser el mismo Isidro Díaz que se casa el 16/5/1747 en el fuerte de San Miguel de Calbuco $^{5}$ con Bartola Mejía (probablemente, la misma que es hija legítima de Juan de Mejía y de Antonia de Soto). Sus hijos conocidos son:

i. Tomasa Díaz. Bautizada de un día en fecha 18/9/1750 en el fuerte de San Miguel de Calbuco, siendo su padrino Diego Rollizo. ${ }^{6}$

- Isidro. Hijo natural de María Bravo y de padre no conocido. Bautizado de catorce días de edad el 29/5/1721 en Potosí, siendo su madrina Petrona Carrasco (LBPs5, foja 90v).

- Isidro. Hijo natural de Magdalena (cuyo apellido no puede leerse con facilidad, aunque parece ser Díaz) y de padre no conocido. Bautizado de seis meses el 25/9/1721 en Potosí, siendo su padrino Domingo Ponce de León (LBPs5, foja 99v).

- Isidro. Expuesto a las puertas de Domingo Barroso. Bautizado de dos meses el 29/7/1723 en Potosí, siendo su madrina D. ${ }^{a}$ Gregoria Salduendo (LBPs5, foja 107v).

3 LMCa1, foja 57v, № 248.

${ }^{4}$ LMCa1, foja 82 , № 343.

${ }^{5}$ LMCa1, foja 39, № 151.

${ }^{6}$ LBCa1, foja $46 \mathrm{v}$. 
ii. Juan Francisco Floriano Díaz, quien sigue la línea.

iii. María Gertrudis Díaz. Bautizada de 15 días el 17/3/1754 en el fuerte de San Miguel de Calbuco, siendo su padrino Miguel Mejía. ${ }^{7}$

iv. Gregorio Casimiro Díaz. Bautizado de caridad a la edad de un mes el 15/6/1559 en el fuerte de San Miguel de Calbuco, siendo su madrina Isabel de Soto. ${ }^{8}$

v. María Isabel Díaz. Bautizada de un mes y medio el 26/7/1761, siendo sus padrinos Juan González y María Isabel Isquerra. ${ }^{9}$

vi. Ignacia Josefa Díaz, llamada usualmente Ignacia Díaz. Natural de Caicaén. Bautizada de quince horas el 31/7/1764 en la iglesia parroquial de Calbuco, siendo sus padrinos Eusebio Aguilar [Iㅇ] y Magdalena de Juanes. ${ }^{10}$ Casada y velados en diciembre de 1793 en el fuerte de San Miguel de Calbuco $^{11}$ con Domingo Rivera (natural de Rulo; hijo legítimo de Pedro Rivera y de Sebastiana González). Parece ser también la misma persona llamada Ignacia Díaz, que tuvo descendencia natural de Eugenio Soto en:

1. Isidro Díaz. Bautizado de tres meses el 13/8/1784, siendo sus padrinos Juan Francisco Floriano Díaz y María Tomasa Guerrero. ${ }^{12}$

\footnotetext{
${ }^{7}$ LBCa1, foja 56.

${ }^{8}$ LBCa1, foja 71.

${ }^{9}$ LBCa1, foja $77 \mathrm{v}$.

${ }^{10}$ LBCa1, foja $85 \mathrm{v}$.

11 LMCa1, foja 183.

12 LBCa1, foja $165 \mathrm{v}$.
} 
vii. Un varón, cuyo nombre no puede leerse por faltar una parte de la foja (que puede ser Gregorio Casimiro Díaz, pero no Juan Francisco Floriano Díaz). Casado y velados en febrero de 1780 en Calbuco ${ }^{13}$ con Cristina Hernández (hija legítima de Cristóbal Hernández de Pascuala Molina).

II. Juan Francisco Floriano Díaz, llamado usualmente Francisco Díaz. Natural del fuerte de Calbuco; bautizado de un mes y medio el 9/7/1752 en el fuerte de San Miguel de Calbuco, siendo su padrino el alférez D. ${ }^{n}$ Diego Rollizo. ${ }^{14}$ Casado y velados el 26/12/1777 ${ }^{15}$ con María Tomasa Guerrero (hija legítima de Martín Guerreros y de Juana Chaves). Al parecer, domiciliados en 1781 en Caicaén. ${ }^{16}$ Con descendencia (i.); parece ser el mismo Francisco Díaz que deja descendencia legítima de Tomasa Alvarado, tal vez variante de Tomasa Guerrero (ii., iii.):

i. Leandro Díaz. Bautizado de seis meses en fecha 9/9/1781, siendo su padrino Cristóbal Hernández. ${ }^{17}$

ii. Guillermo Díaz, quien sigue la Línea 1.

iii. Pablo Díaz, quien sigue la Línea 2.

iv. Cecilio Díaz. Sepultado con entierro menor a la edad de ocho meses de edad, el 12/9/1795 en la parroquial de San Miguel de Calbuco. ${ }^{18}$

\section{Línea 1: Guillermo Díaz}

I. Guillermo Díaz. De la capilla de San José de Calbuco. Sepultado a la edad de cuarenta años el 4/9/1832 en la parroquia de Calbuco. ${ }^{19}$

\footnotetext{
${ }^{13}$ LMCa1, foja $119 v$.

14 LBCa1, foja 49v.

15 LMCa1, foja $117 v$.

${ }^{16}$ LBCa1, foja 156.

17 LBCa1, foja 156.

18 LDCa1, foja 10.

19 LDCa1, foja 208.
} 
Casado y velados el 20/5/1817 en la parroquial de San Miguel de Calbuco ${ }^{20}$ con Mercedes Silva $^{21}$ (hija legítima de Lorenzo Silva y de Nieves Mansilla). Con descendencia:

i. Pascuala Díaz. De la capilla de San José. Casada y velados el 16/9/1841 en la parroquia de San Miguel de Calbuco ${ }^{22}$ con Marcelino Paredes (de la capilla de San Rafael; hijo legítimo de Ignacio Paredes y de Asunciona Oyarzo). Con descendencia.

ii. María Cruz Díaz. Natural y domiciliaria de la doctrina de Calbuco. Casada y velados en

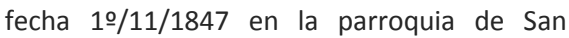
Miguel de Calbuco ${ }^{23}$ con Manuel Soto (natural y domiciliario de la doctrina de Calbuco; hijo legítimo de Leandro Soto y de Antonia Gutiérrez). Con descendencia.

iii. Cayetano Díaz, quien sigue la Línea 1.1.

iv. Agustín Díaz, quien sigue la Línea 1.2.

v. Pedro Díaz. Nacido en 1823 en San José. Sepultado el 12/1/1843 en la parroquia de Calbuco. ${ }^{24}$

vi. José Domingo Díaz. Natural de la capilla de San José y domiciliario de la doctrina de Calbuco. Casado en fecha 15/12/1861 en la parroquia de San Miguel de Calbuco ${ }^{25}$ con Asunción Ojeda (natural de la capilla de San José de Calbuco, y domiciliaria de la doctrina

\footnotetext{
20 LMCa4, foja 14, № 25.

${ }^{21}$ Mercedes Silva (viuda de Guillermo Díaz) es casada y velados con Ramón Paredes (de San Rafael; viudo de Plácida Mansilla; hijo legítimo de Juan José Paredes y de Antonia Velásquez), el 24/11/1834 en la parroquia de San Miguel de Calbuco (LMCa4, foja 260, № 838).

22 LMCa4, foja 360, № 1198.

23 LMCa5, foja 151, № 254.

24 LDCa1, foja 273.

25 LMCa7, foja 126 , № 340.
} 
de Calbuco; hija legítima de Joaquín Ojeda y de Francisca Díaz), siendo dispensados de consanguinidad en segundo grado mixto con tercero por línea transversal desigual.

\section{Línea 1.1.: Cayetano Díaz}

I. Cayetano Díaz. Natural y domiciliario de la doctrina de Calbuco. Casado el 5/12/1853 en la parroquia de San Miguel de Calbuco ${ }^{26}$ con Pascuala Mansilla (natural y domiciliaria de la doctrina de Calbuco; hija legítima de Alejandro Mansilla y de María Juana Alvarado); y luego casado el 2/1/1897 en la capilla de San José de la parroquia de Calbuco ${ }^{27}$ con María Ventura Cárdenas (viuda de José María Nahuelpi; hija legítima de José María Cárdenas y de María Juana Mancilla); quizá luego casado con Candelaria Soto. Con descendencia de por lo menos el primero (i.-iv.) de sus dos matrimonios en:

i. María Flora Díaz. Del curato de Calbuco. Casada en fecha 2/4/1876 en la iglesia parroquial de San Miguel de Calbuco y velados el $13 / 6 / 1877^{28}$ con José Soto (natural y domiciliario de la parroquia de Calbuco; viudo seis meses de Benita Soto; hijo legítimo de Ignacio Soto y de Ignacia Soto), dispensados de un impedimento de afinidad.

ii. Juana Díaz. Natural del curato de Calbuco. Casada y velados gratis el 16/4/1882 en la iglesia parroquial de San Miguel de Calbuco ${ }^{29}$ con Pedro Soto (del curato de Calbuco; fiscal; hijo legítimo de Mariano Soto y de María Benita Velásquez); y casada el 7/11/1893 en la

\footnotetext{
${ }^{26}$ LMCa6, foja 174 , № 307.

27 LMCa11, fojas 71 y 72 , № 2.

${ }^{28}$ LMCa8, foja 29, № 738.

29 LMCa8, foja 171, № 198.
} 
capilla de San Antonio ${ }^{30}$ con Fernando Aguilar (hijo legítimo de José Ignacio Aguilar y de Juana Barría).

iii. Balbina Díaz. Natural y domiciliaria de la parroquia de Calbuco. Casada y velados en fecha 3/9/1885 en la iglesia parroquial de San Miguel de Calbuco ${ }^{31}$ con Manuel Soto ${ }^{32}$ (natural y domiciliario de la parroquia de Calbuco, de la capilla de San José; hijo legítimo de Manuel Soto y de María Cruz Díaz), siendo dispensados de consanguinidad en segundo grado colateral igual.

iv. Francisco Díaz, quien sigue la línea en II.

II. Francisco Díaz. Del curato de Calbuco. Casado el 30/7/1894 en la iglesia parroquial de San Miguel de Calbuco y velados en fecha 17/10/1896 en la iglesia parroquial de San Miguel de Calbuco ${ }^{33}$ con Carmen Barría (del curato de Calbuco; hija legítima de Antonio Barría y de Juliana Sánchez).

\section{Línea 1.2.: Agustín Díaz}

I. Agustín Díaz. Natural de la capilla de San José de Calbuco y domiciliario en la doctrina de Calbuco. Casado el 2/5/1856 en la parroquia de San Miguel de Calbuco ${ }^{34}$ con Candelaria Soto (natural de la capilla de Caicaén y domiciliaria de la doctrina de Calbuco; hijo legítimo de Ignacio Soto y de Ignacia Soto). Con descendencia legítima (i.); debe ser el mismo que, de nombre José Agustín Díaz, deja descendencia natural de Fernanda Paredes (ii.); y de nombre

\footnotetext{
${ }^{30}$ LMCa10, fojas 252 y 253 , № 64 .

31 LMCa9, foja 25, № 533 .

32 Manuel Soto (viudo de Balbina Díaz) es casado con Candelaria Oyarzo (del curato de Calbuco; viuda de Gregorio Ojeda; hija natural de Pascual Oyarzo y de Matilde Frón), el 16/9/1894 en la iglesia parroquial de San Miguel de Calbuco (LMCa10, fojas 296 y 297, № 62).

33 LMCa10, foja 288, № 48.

34 LMCa6, foja 296, № 522.
} 
Agustín Díaz, dejando descendencia también natural de Margarita Paredes (iii.) y de Cecilia Oyarzo (iv.) en:

i. María del Carmen Díaz. Del curato de Calbuco; nacida en 1859. Casada y velados en fecha $5 / 10 / 1875$ en la capilla de Peuque ${ }^{35}$ con Ramón Camilo Soto (natural de la parroquia de Calbuco; hijo legítimo de Francisco Soto y de Mercedes Velásquez).

ii. Felipe Díaz. Natural y domiciliario de la parroquia de Calbuco. Casado y velados de caridad el 27/5/1876 en la iglesia parroquial de San Miguel de Calbuco ${ }^{36}$ con Rosaura Mansilla (natural y domiciliaria de la parroquia y doctrina de Calbuco; hija legítima de Mariano Mansilla y de Castidad Almonacid). Con descendencia en:

1. Ercilia Díaz. Del curato de Cal-buco. Casada y velados en fecha 20/4/1899 en la capilla de San Rafael ${ }^{37}$ con Camilo Oyarzo (del curato de Calbuco; hijo legítimo de Pascual Oyarzo y de Can-delaria Silva), dispensados de consanguinidad cuarto grado con cuarto por línea colateral igual.

2. José María Díaz. Natural y domiciliado en la parroquia de Calbuco. Casado el 27/6/1902 en la iglesia parroquial de San Miguel de Calbuco $^{38}$ con Sara Oyarzun (hija legítima de Juan de Dios Oyarzo y de María Josefa Oyarzo).

\footnotetext{
35 LMCa8, foja 11, № 699.

${ }^{36}$ LMCa8, foja 38, № 459.

37 LMCa12, foja 19, № 29.

${ }^{38}$ LMCa12, fojas 137 y 138, № 44.
} 
iii. Bruno Díaz. Natural de la parroquia de Calbuco. Casado y velados el 22/6/1876 en la parroquia de San Miguel de Calbuco ${ }^{39}$ con Carmen Soto (de la parroquia de Calbuco; hija legítima de Mariano Soto y de María Benita Velásquez).

iv. Cayetano Díaz Oyarzo. Nacido en 1852, residente en la capilla de San Agustín. Casado por palabras de presente en fecha 15/11/1877 en la parroquia de Calbuco ${ }^{40}$ con María Loreto Mansilla, llamada también Loreto Vargas en $1903^{41}$ (de la naturaleza y vecindad de San Agustín; nacida en 1852; hija natural de Manuel Mansilla y de Candelaria Vargas). Con descendencia en:

1. José Abel Díaz. De la capilla de Huito. Casado el 20/2/1897 en la iglesia parroquial de San Miguel de Calbuco $^{42}$ con María Rosa Maripillán (natural de Queilen y avecindada en Huito; hija legítima de Silvestre Maripillán y de María Dominga Cumín).

2. José Díaz. Natural de San Agustín y domiciliado en Hualaihué. Casado y velados el 26/1/1903 en la parroquial de Calbuco ${ }^{43}$ con María del Carmen Marín (natural y domiciliada en la parroquia de Calbuco; hija legítima de Vicente Marín y de Cristina Báez).

39 LMCa8, fojas 42 y 43, № 770.

${ }^{40}$ LMCa8, fojas 66 y 67 , № 67.

${ }^{41}$ LMCa12, foja 152, № 3.

42 LMCa11, foja 89, № 41.

${ }^{43}$ LMCa12, foja 152, № 3. 


\section{Línea 2: Pablo Díaz}

I. Pablo Díaz. De la capilla de San José de Calbuco. Casado y velados el 28/5/1817 en la parroquial de San Miguel de Calbuco ${ }^{44}$ con Mercedes Oyarzun (hija legítima de D. ${ }^{n}$ Francisco Alonso Oyarzun y de Purísima Alvarado). Con descendencia legítima (ii.); es probablemente el mismo Pablo Díaz quien deja descendencia natural de Candelaria Ribera (i.) y Candelaria Cacheru, ${ }^{45}$ también llamada Candelaria Cachi en $1864^{46}$ (iii.), así como descendencia legítima de Antonia Barría (iv., v.) en:

i. María Díaz. Natural de la capilla de San José en Calbuco. Casada primero y velados el $7 / 5 / 1832$ en la parroquia de San Miguel de Calbuco $^{47}$ con Francisco Rollizo (de la capilla de San José de Calbuco; hijo legítimo de Ignacio Rollizo y de Isidora Hernández); y casada el 10/4/1839 en la parroquia de San Miguel de Calbuco ${ }^{48}$ con Juan de Dios Bustamante (de la capilla de Caicaén; hijo legítimo de Juan de Dios Bustamante y de Francisca Silva). Con descendencia de sus dos matrimonios por la que se cuenta incluso una rama establecida en la ciudad de Osorno.

ii. Francisca Díaz. De la capilla de San José de Calbuco. Casada y velados el 17/12/1834 en la parroquia de San Miguel de Calbuco ${ }^{49}$ con Joaquín Ojeda (de la capilla de San José de Calbuco; viudo de Candelaria Guerrero; hijo legítimo de José Antonio Ojeda y de Asunciona Oreta). Con descendencia.

\footnotetext{
44 LMCa4, foja 17, № 31.

45 LMCa4, foja 265, № 859 .

${ }^{46}$ LMCa7, foja 190, № 71.

47 LMCa4, foja 225, № 707.

${ }^{48}$ LMCa4, foja 319, № 1087.

${ }^{49}$ LMCa4, foja 261, № 831.
} 
iii. Dolores Díaz. De Llaicha. Casada en fecha 21/5/1836 en la parroquia de San Miguel de Calbuco y velados en $1839^{50}$ con Domingo Marilicán (de Machil; viudo de María Caico; hijo legítimo de Pascual Maricán y de Pascuala Coli); y casada el 14/3/1864 en la parroquia de San Miguel de Calbuco ${ }^{51}$ con Pedro Linco (de la capilla de Chope y domiciliario de la doctrina de Calbuco; nacido en 1804; viudo de María Juliana Marimañ; hijo legítimo de Segundo Linco y de Paula Llancaburi).

iv. Felipe Díaz, que sigue la línea.

v. José Félix Díaz. Natural y domiciliario de la parroquia de Calbuco. Casado y velados el 21/5/1887 en la iglesia parroquial de San Miguel de Calbuco ${ }^{52}$ con Emilia Soto (natural y domiciliaria de la parroquia de Calbuco; hija legítima de Manuel Soto y de Teresa Ruíz).

II. Felipe Díaz. De la feligresía y naturaleza de Calbuco. Casado de caridad por palabras de presente en fecha 19/3/1879 en la parroquia de Calbuco $^{53}$ con Matilde Flores (de la parroquia y naturaleza de Calbuco; hija natural de Ramón Flores y de Antonia Oyarzún); por segunda vez casado y velados el 15/9/1899 en la iglesia parroquial de San Miguel de Calbuco ${ }^{54}$ con Manuela Zúñiga (natural y domiciliada de la parroquia de Calbuco; hija legítima de Cruz Zúñiga y de Teresa Alvarado); y finalmente casado y velados en fecha 22/11/1903 en la parroquia de Calbuco ${ }^{55}$ con Carmen Zúñiga (natural y domiciliada de la parroquia de Calbuco; hija legítima de Juan de la Cruz Zúñiga y de Teresa Alvarado), siendo dispensados de afinidad lícita.

\footnotetext{
50 LMCa4, foja 265 , № 859.

51 LMCa7, fojas 189 y 190, № 71.

52 LMCa9, foja 104, № 664.

53 LMCa8, fojas 83 y 84, № 53.

54 LMCa12, foja 30, № 51.

${ }^{55}$ LMCa12, foja 182 , № 78.
} 


\section{Otros Díaz presentes en Calbuco}

En la zona en estudio existían otras personas apellidadas Díaz, cuyas noticias se transcriben en este apartado. De entre todos ellos, merecen destacarse dos ramas, provenientes unos de la jurisdicción de Castro y otros de la de Dalcahue.

Agustín Díaz y Agustina Caycamán son padres naturales de: Ventura Díaz (de San José de Calbuco; fiscal en $1879{ }^{56}$ casado primero con Vicencia Villarroel; y casado y velados en fecha 23/7/1835 en la parroquia de San Miguel de Calbuco $^{57}$ con Bárbara Villegas, hija legítima de Juan Francisco Villegas y de Petrona Quelinante; con descendencia de por lo menos el segundo de sus dos matrimonios).

Ventura Díaz y Bárbara Villegas son padres legítimos de: Lorenzo Díaz (natural y domiciliario de la doctrina de Calbuco; casado y velados el 9/1/1869 en San Antonio ${ }^{58}$ con Rosa Mansilla, natural y domiciliaria de la doctrina de Calbuco, hija legítima de Alejandro Mansilla y de Juana Alvarado; con descendencia); Pablo Díaz (de la doctrina y naturaleza de Calbuco; casado el 18/8/1879 en la parroquia de Calbuco ${ }^{59}$ con Candelaria Villegas, de la feligresía y doctrina de Calbuco, hija legítima de Feliberto Villegas y de María Concepción Huenchucheo, dispensados de consanguinidad en segundo con tercer grado por línea transversal desigual; con descendencia); Mercedes Díaz (natural y domiciliaria de la parroquia de Calbuco; casada y velados en fecha 19/5/1884 en la capilla de San Antonio ${ }^{60}$ con Pascual Cárcamo, natural y domiciliario de la parroquia de Calbuco, hijo legítimo de Juan de Dios Cárcamo y de Marta Yáñez).

Lorenzo Díaz y Rosa Mansilla son padres legítimos de: Asunción Díaz (de la capilla de San José; casada el 26/4/1896 en la iglesia parroquial de San Miguel de Calbuco ${ }^{61}$ con Ramón Caipillán, de la capilla de San José, hijo legítimo de Pascual Caipillán y de Petronila Huaiquín); María Dolores Díaz (del

\footnotetext{
56 LMCa8, foja 90, № 75.

57 LMCa4, foja 268, № 862.

58 LMCa7, foja 257, № 26.

59 LMCa8, foja 90, № 75.

60 LMCa8, foja 259, № 412.

61 LMCa11, fojas 24 y 25, № 16.
} 
curato de Calbuco; casada el 7/1/1897 en la capilla de San Antonio ${ }^{62}$ con Manuel Hüirimilla, de la capilla de San José, hijo legítimo de Marcelino Hüirimilla y de Rosaura Hüaiquín); Ventura Díaz (natural y domiciliado en la parroquia de Calbuco; casado el 12/8/1900 en la iglesia parroquial de San Miguel de Calbuco y velados el $17 / 6 / 1902^{63}$ con Adela Oyarzun, natural de Puerto Montt y domiciliada en San Antonio, hija legítima de Carmen Oyarzun y de Antonia Triviño).

Pablo Díaz y Candelaria Villegas son padres legítimos de: Juan Díaz (del curato de Calbuco; casado y velados el 30/1/1899 en la capilla de San José ${ }^{64}$ con Antonia Silva, hija legítima de Vicente Silva y de María Clara Relma); Tomasa Díaz (natural y domiciliada en el curato de Calbuco; casada el 28/3/1900 en la iglesia parroquial de San Miguel de Calbuco y velados en fecha 9/2/1901 en la parroquia de Calbuco ${ }^{65}$ con José Rudecindo Soto, natural y domiciliado en la parroquia de Calbuco, hijo legítimo de Antonio Soto y de Virgilia Cárcamo).

Alonso Díaz (casado y velados el 14/1/1732 en la santa iglesia parroquial del fuerte de San Miguel de Calbuco ${ }^{66}$ con Elena de Igor, también llamada María Igor; con descendencia).

Alonso Díaz y Elena de Igor son padres legítimos de: Ambrosio Díaz (casado y velados en fecha 25/4/1756 en el fuerte de San Miguel de Calbuco ${ }^{67}$ con Lucía Vargas, hija legítima de José Vargas y de Sebastiana Torres; con descendencia); Pedro Díaz (natural de Rilán; casado primero con Basilia Chacón; y casado y velados el 5/6/1805 en la iglesia parroquial de Castro ${ }^{68}$ con María Isabel Sánchez, ${ }^{69}$ hija legítima de Casimiro Sánchez y de Martina Pérez; con descendencia de por lo menos el primero de sus dos matrimonios).

\footnotetext{
62 LMCa11, foja 76, № 12.

63 LMCa12, foja 65, № 42.

${ }^{64}$ LMCa12, foja 16, № 15.

65 LMCa12, foja 51, № 10.

${ }^{66}$ LMCa1, foja 30, № 75.

${ }^{67}$ LMCa1, foja 57, № 245.

68 LMC7, foja 32.

${ }^{69}$ María Isabel Sánchez (viuda de Pedro Díaz) es casada y velados con Antonio Cárcamo (hijo legítimo de Francisco Cárcamo y de Cecilia Calisto), el 22/6/1808 en la iglesia matriz de Castro (LMC7, foja 79v).
} 
Ambrosio Díaz y Lucía Vargas son padres legítimos de: Virginia Díaz (de la isla de Maillén; sepultada con entierro mayor cantado en fecha 28/3/1820 en la parroquial de San Miguel de Calbuco; ${ }^{70}$ casada con José Vargas; con descendencia); Plácida Díaz (de la isla de Puluqui; sepultada con entierro menor rezado el 19/1/1821 en la parroquial de San Miguel de Calbuco; ${ }^{71}$ casada el 2/4 y velados en fecha 19/4/1786 en el fuerte de San Miguel de Calbuco $^{72}$ con Juan Ignacio Gallardo, viudo de Atanasia Igor, hijo legítimo de D. ${ }^{n}$ Ignacio Gallardo y de María Pérez de Bocanegra; con descendencia).

Silvestre Díaz y María Ruíz son padres legítimos de: Andrés Díaz (de Rilán; bautizado el 13/2/1771; casado primero con Rosa Alvarado; y casado y velados el 2/10/1815 en la iglesia matriz de Castro $^{73}$ con Dionisia Cárdenas, ${ }^{74}$ del pueblo de Rilán, hija legítima de Gregorio Cárdenas y de Feliciana Oyarzo; con descendencia de por lo menos el segundo de sus dos matrimonios); JAVIER DíAz (natural de Rilán; casado y velados el 9/6/1806 en la parroquial de Castro $^{75}$ con Prudencia Saldivia, natural de Rauco, hija legítima de Pedro Saldivia y de Isidora Oyarzo; con descendencia).

Andrés Díaz y Dionisia Cárdenas son padres legítimos de: José Díaz (natural de Rilán en la jurisdicción de Castro y avecindado en la doctrina de Calbuco; casado y velados el 8/8/1849 en la iglesia parroquial de San Miguel de Calbuco ${ }^{76}$ con María Jesús Velásquez, natural y domiciliaria de la doctrina de Calbuco, hija legítima de Virginio Velásquez y de Lorenza Vargas; con descendencia).

José Díaz y María Jesús Velásquez son padres legítimos de: María Carmen Díaz (natural y habitante de la capilla de Chope; casada y velados el 4/9/1869 en la capilla de Chope $^{77}$ con Fernando Gallardo, hijo legítimo de Manuel Gallardo y de María Cruz González; con descendencia); Benito Díaz (natural y domiciliario de la capilla de Chope; nacido en 1853; casado el

\footnotetext{
${ }^{70}$ LDCa1, foja $141 v$.

${ }^{71}$ LDCa1, foja 146.

72 LMCa1, foja $127 \mathrm{v}$.

73 LMC8, foja $1 \mathrm{v}$.

74 Dionisia Cárdenas (viuda de Andrés Díaz) es casada con Francisco Trujillo (natural de Rilán; hijo legítimo de Mateo Trujillo y de Narcisa Cárcamo), el 15/10/1835 (LMC9, foja 68, № 241).

75 LMC7, foja 44.

76 LMCa5, fojas 204 y 205, № 363.

77 LMCa7, fojas 288 y 289 , № 84.
} 
6/2/1873 en la iglesia parroquial de San Miguel de Calbuco ${ }^{78}$ con María Aurora Álvarez, natural y domiciliaria de la capilla de Quetrulauquen, nacido en 1857, hija legítima de Aniceto Álvarez y de Mercedes Soto; con descendencia); Primitivo Díaz (natural y domiciliario de la parroquia de Calbuco; casado y velados el 13/11/1880 en la capilla de Machil ${ }^{79}$ con Balbina Gutiérrez, de la parroquia de Calbuco, hija legítima de Ramón Gutiérrez y de Silveria Cárcamo; con descendencia); Gabino Díaz (natural y domiciliario de la parroquia de Calbuco; casado y velados el 11/1/1893 en la iglesia parroquial de San Miguel de Calbuco ${ }^{80}$ con Ignacia Maldonado, natural de Puerto Montt y domiciliaria en la parroquia de Calbuco, hija legítima de Damián Maldonado y de Pedrosa Alvarado).

Benito Díaz y María Aurora Álvarez son padres legítimos de: Juan Francisco Díaz (del curato de Calbuco; casado en fecha 28/6/1894 en la iglesia parroquial de San Miguel de Calbuco ${ }^{81}$ con María Rosa Almonacid, hija legítima de Paulino Almonacid y de María Caipillán); Sandalio Díaz (de la capilla de Chope; casado el 12/5/1897 en la iglesia parroquial de San Miguel de Calbuco $^{82}$ con Isidora Soto, de Reloncaví, hija legítima de Francisco Soto y de Carlina Almonacid).

Primitivo Díaz y Balbina Gutiérrez son padres legítimos de: José Adolfo Díaz (natural y domiciliado en Chope; casado y velados el $7 / 10 / 1901$ en la iglesia parroquial de San Miguel de Calbuco ${ }^{83}$ con María Silveria Guerrero, natural y domiciliada en Quenu, hija legítima de Juan Andrés Guerrero y de María Natividad Núñez).

Javier Díaz y Prudencia Saldivia son padres legítimos de: Cirilo Díaz (natural de la capilla de San Javier; casado y velados el 19/8/1834 ${ }^{84}$ con Antonia Torres, natural de la capilla de Rauco, hija legítima de Pedro Torres y de Rosa Saldivia, dispensados de consanguinidad en segundo grado; y casado y velados el 9/8/1841 en la parroquia de Castro ${ }^{85}$-constando como natural

\footnotetext{
78 LMCa7, foja 484, № 423.

79 LMCa8, foja 125 , № 87.

80 LMCa10, fojas 213 y 214, № 3.

81 LMCa10, fojas 279 y 280 , № 35.

82 LMCa11, foja 92, № 48.

${ }^{83}$ LMCa12, fojas 111 y 112 , № 75.

84 LMC9, foja 48 , № 82.

${ }^{85}$ LMC9, foja 93 bis.
} 
de Rilán- con María Patrocinia Torres, natural de Rilán, hija natural de Nicolás Torres y de Juana Trujillo).

Domingo Díaz (nacido aproximadamente en 1750; de la isla de Puluqui; sepultado con entierro menor rezado el 10/2/1800 en la parroquial de San Miguel de Calbuco; ${ }^{86}$ casado con Martina Igor).

José Díaz y María del Socorro Agüero son padres legítimos de: Lorenzo Díaz (natural de la capilla de Alfaro y domiciliario de la doctrina de Calbuco; nacido en 1839; casado el 23/7/1867 en la parroquia de San Miguel de Calbuco ${ }^{87}$ con Tránsito Ruíz, natural de la capilla de Alfaro y domiciliaria de la doctrina de Calbuco, hija legítima de Francisco Ruíz y de Tomasa Hernández; con descendencia); Candelaria Díaz (natural y domiciliaria de la capilla de Alfaro; nacida en 1839; casada de caridad el 24/2/1874 en la iglesia parroquial de San Miguel de Calbuco ${ }^{88}$ con Vicente Yáñez, natural y domiciliario de la capilla de Alfaro, hijo legítimo de Antonio Yáñez y de Benigna Almonacid).

Lorenzo Díaz y Tránsito Ruíz son padres legítimos de: María Domicinda Díaz (natural de Guar y domiciliaria en Rolecha; casada y velados el 12/6/1896 en la iglesia parroquial de San Miguel de Calbuco $^{89}$ con Jacinto Mancilla, de Rolecha, hijo legítimo de Inocencio Mancilla y de Vicencia Llancapani).

Juan Díaz y Saturnina Vargas son padres naturales de: José Díaz (casado y velados de caridad en fecha 26/5/1876 en la iglesia parroquial de San Miguel de Calbuco ${ }^{90}$ con Nicolasa Villegas, hija legítima de Miguel Villegas y de Rosa Silva; con descendencia legítima, como probablemente también con descendencia natural de Emilia Vargas).

José Díaz y Nicolasa Villegas son padres legítimos de: Pedro Manuel Díaz (del curato de Calbuco; casada y velados el 30/1/1899 en la capilla de San José ${ }^{91}$ con Rosa Guerrero, del curato de Calbuco, hija legítima de Valentín Guerrero y de Josefa Soto); Ignacio Díaz (del curato de Calbuco;

\footnotetext{
${ }^{86}$ LDCa1, foja 34.

${ }^{87}$ LMCa7, foja 223, № 144.

88 LMCa7, fojas 552 y 553 , № 532.

89 LMCa11, fojas 34 y 35, № 36.

90 LMCa8, foja 36, № 756.

91 LMCa12, foja 16, № 16.
} 
casado y velados de caridad el 26/5/1876 en la iglesia parroquial de San Miguel de Calbuco ${ }^{92}$ con Leonor Soto, del curato de Calbuco, hija legítima de Pascual Soto y de Tránsito Calisto).

José Díaz y Emilia Vargas son padres naturales de: Delfina Díaz (natural y domiciliada en San José; casada y velados el 12/9/1901 en la parroquia de Calbuco $^{93}$ con Pedro Ñanco, natural y domiciliado en la parroquia de Calbuco, hijo legítimo de Marcelino Ñanco y de Candelaria Ñanco).

Pedro Díaz (casado con María Agustina Mansilla; ${ }^{94}$ con descendencia). Pedro Díaz y María Agustina Mansilla son padres legítimos de: José Díaz (natural y domiciliario de la parroquia de Calbuco; casado y velados de caridad el 22/1/1887 en la iglesia parroquial de San Miguel de Calbuco ${ }^{95}$ con María Belisaria Velásquez, natural y domiciliaria de la parroquia de Calbuco, hija legítima de Narciso Velásquez y de Bernarda Vargas).

Pedro María Díaz (casado con Manuela Pérez, ${ }^{96}$ hija legítima de Antonio Pérez y de Rosa Barrientos; con descendencia).

Pedro María Díaz y Manuela Pérez son padres legítimos de: Juan Bautista Díaz (natural del departamento de Dalcahue y habitando en la capilla de Tabón; casado y velados el 6/2/1853 en la parroquia de San Miguel de Calbuco ${ }^{97}$ con María Nicolasa Agüero, hija legítima de Santiago Agüero y de María Castidad Higuera); Rosario Díaz (de la capilla de Tabón y domiciliaria de la doctrina de Calbuco; casada el 19/5/1855 en la parroquia de San Miguel de Calbuco ${ }^{98}$ con Juan José Agüero, hijo legítimo de Santiago Agüero y de María Castidad Higuera; con descendencia); Luis Díaz (natural de la capilla de Tabón y domiciliario de la doctrina de Calbuco; casado en fecha 24/3/1856 en la

\footnotetext{
92 LMCa12, foja 16, № 17.

93 LMCa12, foja 105, № 59

94 María Agustina Mansilla (viuda de Pedro Díaz) es casada de media caridad con Hilario Barquero (natural y domiciliario de la doctrina de Calbuco; nacido en 1841; hijo natural de María Barquero y de padre no conocido), el 20/4/1870 en la parroquia de San Miguel de Calbuco (LMCa7, foja 312, № 130).

95 LMCa9, foja 89, № 639.

96 Manuela Pérez (viuda de Pedro María Díaz) es casada con Tertuliano Vargas (natural de Calbuco; fiscal; viudo de Teresa Alvarado), el 3/11/1844 en la capilla de Dalcahue (LMT1, foja 52).

97 LMCa6, fojas 129 y 130, № 224.

98 LMCa6, fojas 245 y 246 , № 434.
} 
iglesia parroquial de San Miguel de Calbuco ${ }^{99}$ con Dolores Soto, natural de la capilla de Caicaén y domiciliaria de la doctrina de Calbuco, hija legítima de José María Soto y de María Rosa Soto); Marcelino Díaz (natural de la capilla de Dalcahue y residente en la capilla de Caicaén; bautizado de caridad a la edad de dos años, por Pedro Gallardo, el 8/11/1843 en Dalcahue, siendo sus padrinos Antonio Muñoz y Catalina Gallardo; ${ }^{100}$ casado en fecha 7/8/1865 en la iglesia parroquial de San Miguel de Calbuco ${ }^{101}$ con María Soto, natural de la capilla de Caicaén, hija legítima de José María Soto y de Tomasa Soto; con descendencia).

Marcelino Díaz y María Soto son padres legítimos de: Carmen Díaz (del curato de Calbuco; casada el 2/4/1891 en la iglesia parroquial de San Miguel de Calbuco ${ }^{102}$ con José Ignacio Almonacid, natural y domiciliario de Puerto Montt, viudo de María Carlota Velásquez, hijo legítimo de Francisco Almonacid y de María Inés Soto); Rosario Díaz (natural y domiciliada en el curato de Calbuco; casada y velados el 16/6/1902 en la capilla de San Antonio $^{103}$ con Luis Gallardo, hijo legítimo de Nicolás Gallardo y de Bonifacia Bustamante).

Roque Díaz y Pascuala Mansilla son padres legítimos de: María Nieves Díaz (de la capilla de Quetrulauquen; casada y velados el 5/4/1842 en la parroquia de San Miguel de Calbuco ${ }^{104}$ con Francisco Olabarría, hijo legítimo de Cayetano Olavarría y de Antonia Almonacid; y casada en fecha 12/11/1854 en la parroquia de San Miguel de Calbuco ${ }^{105}$ con Vicente Soto, natural y domiciliario de la doctrina de Calbuco, hijo natural de José Soto y de Encarnación Garay).

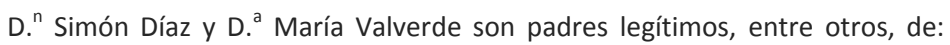
D. ${ }^{N}$ Fernando Díaz (casado y velados el 15/11/1748 en la ciudad de Castro ${ }^{106}$ con D. ${ }^{a}$ Francisca Mansilla, hija legítima de D. ${ }^{n}$ Francisco Mansilla y de D. ${ }^{a}$ Beatriz Barrientos; con descendencia).

\footnotetext{
99 LMCa6, foja 291, № 511.

100 LBT1, foja 71.

101 LMCa7, foja 201, № 98.

102 LMCa10, foja 127, № 989.

103 LMCa12, foja 134, № 35.

104 LMCa4, foja 370, № 1248.

105 LMCa6, fojas 227 y 228 , № 407.

106 LMC2, foja 48.
} 
D. ${ }^{n}$ Fernando Díaz y D. ${ }^{a}$ Francisca Mansilla son padres legítimos de: D. ${ }^{a}$ María Mercedes Díaz (casada y velados el 31/10/1781 ${ }^{107}$ con D. ${ }^{n}$ Tomás Pérez, ${ }^{108}$ viudo de D. ${ }^{a}$ María Zarauz, hijo legítimo de D. ${ }^{n}$ Justo Pérez y de D. ${ }^{a}$ Nicolasa Pérez de Aguilar, previa dispensa de "3.o gr. ${ }^{\circ}$ mixto con 4. de sang. ${ }^{d}$ y en 3. o de afinidad"; con descendencia); D. N Feliberto Díaz (casado primero en fecha $28 / 7 / 1783^{109}$ con D. ${ }^{a}$ Rosa Andrade, viuda de D. ${ }^{n}$ Ignacio Mayz, hija legítima de D. ${ }^{n}$ Ignacio Patricio Andrade y de D. ${ }^{a}$ Juana Vargas, dispensados de cuarto grado y de las amonestaciones; y casado con $\mathrm{D}$. $^{\mathrm{a}}$ Bonifacia Mansilla, hija legítima de D. ${ }^{n}$ Manuel Mansilla y de D. ${ }^{a}$ Josefa Miranda; con descendencia de sus dos matrimonios, como con descendencia natural de Policarpa Agüero); D. a Asunta Díaz (casada y velados en fecha $21 / 8 / 1783^{110}$ con D. ${ }^{n}$ Juan Bautista Bórquez, hijo legítimo de D. ${ }^{n}$ Juan Bautista Bórquez y de D.a Manuela Cárcamo; con descendencia).

D. ${ }^{n}$ Feliberto Díaz y D. ${ }^{a}$ Rosa Andrade son padres legítimos de: Vicente Díaz (bautizado el 5/9/1784); Rafaela Díaz (bautizada el 25/10/1785);

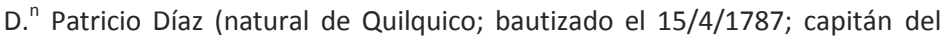
Ejército Real, asistente a las batallas de Mocopulli, Pudeto y Bellavista, y firmante del tratado de Tantauco; ${ }^{111}$ casado y velados el 21/6/1806 en la iglesia de San Francisco en Castro ${ }^{112}$ con D. ${ }^{a}$ Rosa Arteche, hija legítima de D. ${ }^{n}$ José Arteche y de D. ${ }^{a}$ Aurelia Andrade; y casado y velados el 24/5/1836 en la iglesia parroquial de Castro ${ }^{113}$ con D. ${ }^{a}$ Rosario Cárdenas, hija legítima de D. ${ }^{n}$ Luis Gonzaga Cárdenas y de D. ${ }^{a}$ Ignacia Castellón; con descendencia de sus dos matrimonios).

D. ${ }^{n}$ Feliberto Díaz y Policarpa Agüero son padres naturales de: D. ' José María Díaz (natural de la capilla de Chonchi; casado y velados en

\footnotetext{
107 LMC4, foja $104 \mathrm{v}$.

108 D. ${ }^{\text {n }}$ Tomás Pérez (viudo de D. ${ }^{a}$ María Zarauz) es casado con Josefa Montiel (natural de Quilquico; hija legítima de Miguel Montiel y de Valeriana Silva), el 24/3/1805 en la iglesia parroquial de Castro (LMC7, foja 26v).

109 LMC4, foja $118 \mathrm{v}$.

110 LMC4, foja $119 v$.

111 Guarda, página 144

112 LMC7, foja 44v.

113 LMC9, foja $25 v$ bis.
} 
fecha 9/11/1813 en la iglesia de Chonchi ${ }^{114}$ con D. ${ }^{a}$ Cristina Oyarzun, hija legítima de D. ${ }^{n}$ Mariano Oyarzun y de D. ${ }^{a}$ Juana Gómez).

D. ${ }^{n}$ Feliberto Díaz y D. ${ }^{a}$ Bonifacia Mansilla son padres legítimos de: D. ${ }^{n}$ José María Díaz, también llamado D. ${ }^{n}$ José Domingo Díaz en $1847^{115}$ (natural de Putemún; casado y velados el 11/7/1817 en la iglesia matriz de Castro $^{116}$ con D. ${ }^{a}$ María Rita Garay, hija legítima de D. ${ }^{n}$ Antonio Ramón Garay y de D. ${ }^{a}$ Marta Loayza; con descendencia); D. ${ }^{a}$ Dolores Díaz (casada y velados el 21/8/1816 en la matriz de Castro ${ }^{117}$ con D. ${ }^{n}$ José Ignacio Andrade, de Yutuy, hijo adoptivo de D. ${ }^{n}$ Basilio Andrade y de D. ${ }^{a}$ Juana Pérez, dispensados de consanguinidad en cuarto grado); Manuela Díaz (bautizada el 28/1/1789); D. ${ }^{a}$ Manuela Díaz (bautizada el 28/12/1800; natural de Putemún; casada y velados en fecha 27/6/1819 en la iglesia matriz de la ciudad de Castro con D. ${ }^{n}$ Antonio Bórquez, natural de Quilquico, hijo legítimo de D. ${ }^{n}$ Valeriano Bórquez y de D. ${ }^{a}$ Josefa Cárdenas); D. ${ }^{a}$ Rufina Díaz (natural de Putemún; casada el 7/7/1825 $5^{118}$ con D. ${ }^{n}$ Francisco Pérez, hijo legítimo de D. ${ }^{n}$ Cosme Pérez y de D. ${ }^{a}$ Andrea Díaz; ${ }^{119}$ con descendencia).

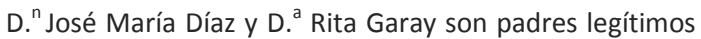
de: D. ${ }^{n}$ José Agustín Díaz (natural de Castro y domiciliario de la doctrina de Calbuco; casado en fecha 28/10/1847 en la parroquia de San Miguel de Calbuco $^{120}$ con D. ${ }^{a}$ María del Carmen González, natural y domiciliaria de la doctrina de Calbuco, hija legítima de D. ${ }^{n}$ Cayetano González y de D. ${ }^{a}$ Rosa Alvarado; con descendencia).

D. ${ }^{n}$ José Agustín Díaz y D. ${ }^{a}$ María del Carmen González son padres legítimos de: D. ${ }^{n}$ Eduardo Díaz (natural de Calbuco y domiciliario en la parroquia de Ancud; casado primero con Candelaria Soto; y casado en fecha 20/6/1883 en la iglesia catedral de Ancud ${ }^{121}$ con Victoria Cárdenas, natural y domiciliaria de la parroquia de Ancud, hija legítima de Juan Bautista Cárdenas y de Victoria Navarro).

\footnotetext{
114 LMC7, foja 165.

115 LMCa5, foja 150, № 252.

116 LMC8, foja 33.

117 LMC8, foja 16.

118 LMC8, foja 196.

119 Pérez, página 302.

120 LMCa5, foja 150, № 252.

121 LMAn1, foja 151, № 368.
} 


\section{Documentación}

Las siglas utilizadas en este escrito corresponden a las siguientes fuentes documentales:

LBCa1: Volumen sin tapas, portada ni título alguno. Una hoja impresa dice al inicio: "ARCHIVO / DE LA / Secretaría Episcopal / de Puerto Montt / DEP. Calbuco № 1 / 1724-1794 / Libro de Bautismos". La foja 107 no existe (en vez de la cual está la foja 108, numeración que se sigue); la foja 113 está perdida; las 126 y 127 son inexistentes; y las fojas 141-144 están faltantes. Entre las fojas 153 y $153 v$ hay dos carillas en blanco. Luego, a partir de la foja 191 (incluida) no aparece la numeración, que reaparece en la foja 195. Entre las fojas 205v y 206 hay un papel en blanco pegado, y luego hay otro más a continuación del primero; ambos conteniendo asientos.

LBPs3: Este volumen corresponde al libro de bautismos de Potosí № 3, y en su contratapa interna se lee: “№ 3".

LBPs5: Este volumen corresponde al libro de bautismos de Potosí № 5. En el lomo puede leerse: "1718 / 1723", y más abajo, en tinta negra: “5”.

LBPs9: Este volumen corresponde al libro de bautismos de Potosí № 9. En la portada puede leerse, bajo una cruz patada afinada centrada en la parte superior: "Libro, de Baptismos. / En que se Asientan Las Partidas. / de los Baptizados Españoles, En, esta Sanc= / ta iglesia Matriz dela Villa Imperial DE / Pottosi Que se Empeso En VeintiSiete de / abril de 1691 años // Numero 9".

LBT1: Portada: "Libro $1 .^{r o}$ en que / se anotan las partidas / de / oleos / Principia à primero de Enero de / 1838", y más abajo "Non sunt aondione passiones hujus / temporis ad futuram que revela- / voltu in [¿்]novia[?]-S. Pablo.".

LDCa1: Portada: "Livro de Entierros de espa- / ñoles, y Yndios hecho p. ${ }^{r}$ el Cu- / ra autual D." Ramon Camilo / de Lorca, que corre desde / el Año de 1794.". Tapa: "Entierros / de Españoles // Entierros // Entierros"; y más arriba, a la derecha, una estampilla posterior pegada que dice "1794-1844".

LMAn1: Volumen sin portada ni títulos; que dice en el lomo: "MATRIMO / NIO / L. / / / 1879 / a / 1888". Contiene índice.

LMC2: En la portada dice: "Libro En que se [asi-] / entan los Casamientos / de Españoles; El qual Corre / desde primero de Mayo de este año de / mil 
setecietnos y veinte y ocho: Echo / por el Vic. ${ }^{\text {or }} D .^{n}$ Thomas Nieto dela Thorre Cura / y Vicario que fue dela Yglecia de N. ${ }^{a}$ S: ${ }^{a}$ de Guía / çita en el fuerte de $S .^{n}$ Miguel de Calbuco / Y dos vesses Cura en esta de $N .{ }^{a} S^{a}{ }^{a}$ delas / Nieves dela Ciudad de Castro; Yesta / ultima ves; siendo Obispo dela $S^{\text {ta. }}$ / Yglecia dela Ciudad dela Puris. ${ }^{a} /$ Concep. ${ }^{n}$ Y Obispado Imperial / el Ylt. ${ }^{m}$ y R. $^{\text {mo }}$ S. ${ }^{r}$ D. ${ }^{r}$ D. ${ }^{n}$ / Fran. ${ }^{\text {co }}$ Antonio [Esc]andon / del Concejo de su Mag. ${ }^{d}$ / Año de 1728". La foja $1 v$ es verdaderamente la portada del libro en sí.

LMC4: En la portada dice: "Libro en que se a Sientan los Ca / Samientos de los Españoles. el qual / Corre desde Catorse de Marzo / de mil setecientos sesenta / y un años echo por el Mrõ. / D. ${ }^{n}$ Juan Jph de Vera Cu / ra Rector y Vicario actual / que es de Esta Santa Ygle / cia de Nrã. Señora de / las Nieves siendo Go / vernad'. del Obispado / el S. ${ }^{\text {or }} D .^{r} D .^{n}$ Juan / de Gusman y Peral / ta dean de dha. / Santa Yglecia / Año. de 1761; / (con otra grafía:) Año del 1761", cada renglón centrado. En la tapa dice "LIBRO / DE CASAMIENTOS"; y, en un retazo de papel pegado puede leerse: "Lib[ro de] / C[asamien]tos / 176[1-179]0", y arriba a la derecha: "№ 4". La portada debe contarse como foja 1, mientras que la $1 \mathrm{v}$ se encuentra en blanco; y hay un error de numeración, ya que la foja 128 no existe, en vez de la cual está la número 130, numeración que se sigue.

LMC7: Tapa: "LIBRO / Dela Parroquia de Sant. ${ }^{\circ}$ de / Castro enla Prov. deChiloé / al Cargo de su Cura Propi[e-] / tario D. ${ }^{n}$ Fran. ${ }^{\text {co }}$ Xav. ${ }^{r}$ Vene[gas] / [ilegible]ta. / Casa[miento]sde el / [Mar]zo [de] / 1803"; todo sobre lo cual se encuentra un papel blanco pegado, donde hay manuscrito en cursiva: "Libro de 7o / Casamiento[s] / 1803_[1815]". La foja 154 no existe, en vez de la cual existe la 155, que es la numeración que se sigue; y lo mismo sucede con la 190 que no existe, en vez de la cual sigue la 191.

LMC8: Portada: "LIBRO / PAROquial de Santiago de / Castro de los Casamientos que se van / apuntando desde veinte,ytres de Septi- / embre del año de 1815 ", tras lo cual puede leerse, en un papel blanco pegado en el centro, y manuscrito: "[en el extremo superior derecho:] N.o 8 // Libro de / Casamientos. / 1815_1826". Lomo: sin leyenda. Hay un error de numeración, ya que luego de la foja 129v, existe una segunda "129" (Ilamada 129 bis), con su correspondiente $129 \mathrm{v}$ bis; tras lo cual prosigue la numeración en 130.

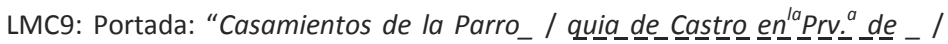
Chiloè Año de: 1826". Tapa: "Libro de matrimonio[s] / [ilegible] capilla 
de Castro / Año de 1826"; sobre lo cual puede leerse, pegado en un papel blanco: "Libro de Matrimonios / 1826 á 1845. // N. o 9-". Lomo: “IX / Matrimonio". Hay errores de numeración: la foja 3 no existe, la cual está llamada 4 (que es la numeración que se sigue); las fojas 29 y $29 \mathrm{v}$ tampoco existen (de manera que luego de la foja $28 \mathrm{v}$ sigue el libro en la $30)$; y luego de la foja $79 \mathrm{v}$, recomienza la numeración en la 20 bis. En la foja 2 hay dos partidas Ilamadas 494, denominadas "494" y "494bis".

LMCa1: Volumen sin portada, ni títulos; pero que tiene en su inicio una hoja impresa, y pegada en el siglo XX, que dice: "ARCHIVO / DE LA / Secretaría Episcopal / de Puerto Montt / DEP. Calbuco N.o 1 / Casamientos de Españoles 4-V-1710- / [en subíndice, bajo la fecha anterior:] 28-II-1770 / Casamientos de Indios 7-VI-1710-18-III-1765 / Casamientos 10-VII-1771 XII-1793". El libro posee dos numeraciones distintas; pero se sigue sin embargo la que parece ser más moderna, que comienza desde la foja primera, numerándose las carillas derechas. La foja $86 \mathrm{v}$ es una carilla en blanco.

LMCa4: Portada, que es a su vez la foja 1: “(1.) / LBBRO 3.\% / de Casamients / de Españoles / y de Yndios / hecho en / el año / De / 1816", todo ello dentro de un triángulo con su vértice más agudo hacia abajo. Una foja antes se encuentra impreso lo siguiente: "ARCHIVO / DE LA / Secretaría Episcopal / de Puerto Montt / DEP. Calbuco N.o 4 / Libro de Casamientos / de Españoles e Indios / 12- Set.1816 - 8-Marzo 1845". Existe un error de numeración de asientos, que pasa del número 580 (en la foja 196) a la 561 (foja 197). Tampoco existen las fojas 226-228, pasándose de la 225 a la 229.

LMCa5: Tapa: "Matrimonios. / Libro 10 / 1889-1895. / J. Krautz / C. y V.". Portada: "Libro 10 de Matrimonios / que principia el 13 de / febrero de 1889; / Máuser". Hay un error de numeración de las partidas, y el asiento 180 de la página 118 se llama 180 bis.

LMCa6: Tapa: "Libro II / De Los / Matrimonios / 1850 a 1857". Portada: "Sigue el / Año 1850. Agosto / En que se escriben / las partidas de casami / de esta Jglecia Parro / quial de San Migu / el de Calbuco, el cual / comienza á correr el / dia treinta i uno de / Agosto del año de mil / ochocientos cincuenta".

LMCa7: Portada: "Libro / en que se escriven las / partidas de Matrimo- / nio de esta iglesia Pa- / rroquial de S. Miguel / de Calbuco que da prin- / cipio el 10 de Junio del / año de mil ochocientos / cincuenta i siete", y abajo un sello que dice: "PARROQUIA SAN MIGUEL DE CALBUCO". Tapa: 
"Libro 70 // Libro de Matrimonios / de la parroquia de S. Miguel / de Calbuco.". Lomo: "L-7 / de 7 / Matrimonios / 1857 á 1873". Luego de la foja 91, vuelve a repetirse una llamada 90 (que se nombra 90 bis) y otra a continuación llamada 91 (que se nombre 91 bis). Faltan las fojas 381 y 382.

LMCa8: Portada, arrancada. Tapa: "Libro 8. // Matrimonios.". Lomo: "8".

LMCa9: Portada: "Libro 9. o de Matrimonios / que principia el 13 de / febrero de 1885. / [firmado:] Mánses". Tapa: "Matrimonios / Libro 10 / 1889-1895. / [firmado:] J. Krautz".

LMCa10: Portada: "Libro 10 de Matri- / monios, que prin- / cipia el 8 de Febrero / de 1885. / [firmado:] Luis Téllez Oyarzun / C. i V.". Tapa: "Libro $9^{\circ} \mathrm{de} /$ Matrimonios / 1885.".

LMCa11: Portada: "Libro 11 / de Matrimonios / 1895- / Calbuco, junio 13 de 1895 / [firmado:] Francisco Krautz / Párroco". Tapa: "Matrimonios / Libro 11 / 1895-1898. / [firmado:] Francisco Krautz / C. i V.".

LMCa12: Portada: "Este libro comienza con la partida de / Crisantos Montiel n.o 46. / [firmado: ilegible]". Tapa: "Matrimonios / Libro 12. / 1898-1899-1901-1902.".

LMT1: Portada: "Libro que, se Sien_ / tan las partidas / de Casamientos que em_ / piensa en 1o de Eenero / de 1838. Cabesera de Tenaun.", y más arriba dice, en la misma portada: "Libro - 1ㅇ”. La foja 70 no existe, y en vez de ella aparece la 80 , de donde recomienza la numeración. Además, entre las fojas $18 \mathrm{v}$ y 19 hay una sin numerar.

\section{Bibliografía citada}

ALCEDO, Don Antonio de. 1788. Diccionario geográfico-histórico de las Indias Occidentales ó América: es á saber: de los Reynos del Perú, Nueva España, Tierra-Firme, Chile, y Nuevo Reyno de Granada. CON LA DESCRIPCION de sus Provincias, Naciones, Ciudades, Villas, Pueblos, Rios, Montes, Costas, Puertos, Islas, Arzobispados, Obispados, Audiencias, Virreynatos, Gobiernos, Corregimientos, y Fortalezas, frutos y producciones; con expresion de sus Descubridores, Conquistadores y Fundadores; Conventos y Religiones; ereccion de sus Catedrales y Obispos que ha habido en ellas: Y NOTICIA de los sucesos mas notables de varios lugares: incendios, terremotos, sitios, é invasiones que han experimentado; y hombres ilustres que han producido. Tomo IV. Madrid: Imprenta de Manuel González. 
ARIAS, Fabián. 2002. “Los pueblos del noroeste de la Patagonia argentina. Relaciones históricas y etnológicas con el sur continental chileno y el Archipiélago de Chiloé. Siglos XVII-XVIII". Revista de Historia 9: 45-70. Neuquén: Universidad Nacional del Comahue.

[http://revele.uncoma.edu.ar/htdoc/revele/index.php/historia/article/view/ 252/238] página descargada el 20 de octubre 2014.

ARZÁNS DE ORSÚA Y VELA, Bartolomé. 2000. Relatos de la Villa Imperial de Potosí: Antología. Selección, introducción y cronología de Leonardo García Pabón. Colección Letras Fundacionales. La Paz: Plural Editores.

COLE, Jeffrey A. 1985. The Potosi Mita, 1573-1700: Compulsory Indian Labor in the Andes. California: Stanford University Press.

GISBERT, Teresa. 2001. "Producción cultural en el mundo andino". Historia de América andina, tomo 3. Margarita Garrido, ed. Quito: Universidad Andina Simón Bolívar. 247-76.

GUARDA, Gabriel, OSB. 2002. Los encomenderos de Chiloé. Santiago: Ediciones Universidad Católica de Chile.

MIRA DELLI-ZOTTI, Guillermo. 1997. “El Real Banco de San Carlos de Potosí y la minería altoperuana colonial”. La savia del Imperio: tres estudios de economía colonial. Julio Sánchez Gómez, Guillermo Mira Delli-Zotti y Rafael Dobado. Salamanca: Ediciones Universidad de Salamanca. 265-350.

MORALEDA I MONTERO, José de. 1888. Exploraciones geográficas e hidrográficas. Comentado por Diego Barros Arana. Documentos para la Historia de la Náutica en Chile. Santiago: Imprenta Nacional.

NACIONES UNIDAS. 1950. La inmigración en Chile (E/CN.12/169/Add.2). Montevideo: Consejo Económico y Social, CEPAL (Comisión Económica para América Latina y el Caribe). Documento presentado en el tercer período de sesiones de la CEPAL (Santiago de Chile 1 de mayo: Santiago de Chile; 5-21 junio 1950: Montevideo).

NAVARRO GARCÍA, Luis. 1983. América en el siglo XVIII. Los primeros borbones. Tomo XI-1. Madrid: Ediciones Rialp, Orymu.

ORDÓÑEZ DE CEBALLOS, Pedro. 1905 [1614]. "Viaje del mundo". Autobiografías y memorias coleccionadas é ilustradas por Manuel Serrano y Sanz. Nueva Biblioteca de Autores Españoles. Madrid: Librería Editorial de Bailly Baillière e Hijos. 270-460.

OTERO, Gustavo Adolfo. 1989. "Estampas bolivianas". Viajeros hispanoamericanos. Estuardo Núñez, ed. Caracas: Biblioteca Ayacucho. 469-83. 
PÉREZ, Pablo A. 2010. "Álvarez en Chiloé, o una rama genealógica no filiada del linaje Bahamonde". Revista de Estudios Históricos LIX (52): 215-276. Santiago: Instituto Chileno de Investigaciones Genealógicas.

PÉREZ HERRERO, Pedro. 1992. Comercio y mercados en América Latina colonial. Madrid: Editorial MAPFRE.

PINO MANRIQUE, Juan del. 1836 [1787]. "Descripción de la villa de Potosí y de los partidos sugetos a su intendencia". Colección de obras y documentos relativos a la Historia Antigua y Moderna de las provincias del Río de la Plata, ilustrados con notas y disertaciones por Pedro de Angelis. Tomo Segundo. Buenos Aires: Imprenta del Estado.

PNUD. 2004. Informe de Desarrollo Humano en Santa Cruz. La Paz: Plural Editores.

SALAZAR-SOLER, Carmen. 2002. "La Villa Imperial de Potosí, cuna del mestizaje (siglos XVI-XVII)". Colonización, resistencia y mestizaje en las Américas. Siglos XVI-XX. Guillaume Boccara, ed. Lima/Quito: Institut français d'études andines IFEA/Editorial Abya-Yala. 139-60.

SILVA Y MOLINA, Abraham de. 1899. Historia de la provincia de Chiloé bajo la dominación española. Obra inédita existente en el Archivo Nacional de Chile.

TANDETER, Enrique. 1992. Coacción y mercado. La minería de la plata en el Potosí colonial, 1692-1826. Madrid: Siglo Veintiuno de España Editores.

TAPIA MONTECINOS, Lourdes; Ernesto Quintana Campana; Delfín Ance Flores, Jenny Morales Cárdenas. 2002. Calidad de vida en Potosí: Efectos ambientales en cuatro zonas del municipio. La Paz: ISALP. Fundación PIEB.

TAULLARD, Alfredo. 2004. Platería sudamericana. Sevilla: Ediciones Espuela de Plata.

\section{$(\mathrm{cc}) \mathrm{BY}$}

\section{ULIS D gate}

New articles in this journal are licensed under a Creative Commons Attribution 4.0 United States License.

This journal is published by the University Library System of the University of Pittsburgh as part of its D-Scribe Digital Publishing Program, and is cosponsored by the University of Pittsburgh Press. 